\title{
Weight Supported Treadmill Ambulation in a Patient with Severe Congestive Heart Failure: A Case Report
}

\author{
Safanah Siddiqui ${ }^{1}$, Muzammil Rehman ${ }^{1}$, Jonathan Daich ${ }^{1}$, John G Kral ${ }^{3}$, Louis Salciccioli ${ }^{1}$, Ellen Godwin ${ }^{2}$ and Jason M \\ Lazar $^{1 *}$ \\ ${ }^{1}$ Division of Cardiovascular Medicine, State University of New York Downstate Medical Center, USA \\ ${ }^{2}$ Human Performance Laboratory, State University of New York Downstate Medical Center, USA \\ ${ }^{3}$ Department of Surgery and Medicine, State University of New York Downstate Medical Center, USA \\ Received: 畊 August 15, 2018; Published: \\ *Corresponding author: Jason M. Lazar, Director, Non-Invasive Cardiology, Downstate Medical Center, State University of New York, \\ 450 Clarkson Avenue, MSC 1199, Brooklyn, New York, USA
}

\begin{abstract}
We describe a 43-year old man with congestive heart failure (CHF) with markedly reduced ejection fraction and New York Heart Association Class 3 to 4 symptoms. After a 6 -minute walk test showed a $140 \mathrm{~m}$ distance, he performed 6 weeks of walking sessions on a weight-supporting lower body positive pressure treadmill (LBPP). Unloading $40 \%$ of his body weight he was able to progressively increase the duration of the walking sessions, exhibited a post-session diuretic effect and dramatically increased his exercise capacity on a 6-minute walk test. He rated his satisfaction with the sessions as maximal. His functional class improved to class 2, remaining stable at 1-year follow-up. We conclude that repeated sessions of ambulation on a weight-supporting LBPP treadmill may benefit patients with CHF with reduced ejection fraction and poor functional capacity.
\end{abstract}

Keywords: Weight Supported Treadmill; Heart Failure; Lower Body Positive Pressure; Six Minute Walk Test

Abbreviations: CHF: Congestive Heart Failure; LBPP: Lower Body Positive Pressure

\section{Introduction}

We describe a 43-year old man with congestive heart failure (CHF) with very low functional capacity who demonstrated a marked improvement in 6-minute walk distance and functional class after 6 weeks of walking sessions on a weight support treadmill.

\section{Case}

The patient is a 43-year old African American overweight man with a long history of hypertension who presented 3 years ago with signs and symptoms of congestive heart failure (CHF). Echocardiography revealed a left ventricular ejection fraction of $25 \%$ and coronary angiography exhibited normal coronary anatomy. He received an implantable cardio-defibrillator and was treated with carvedilol $25 \mathrm{mg}$ bid, enalapril $20 \mathrm{mg}$ qd, furosemide $40 \mathrm{mg}$ qd, spironolactone $25 \mathrm{mg}$ qd and fixed-dose hydralazine 37.5 mg-isosorbide dinitrate $20 \mathrm{mg}$ tid. However, symptoms of shortness of breath and fatigue progressed to occur on minimal exertion and occasionally at rest. On physical exam, body mass index was $29.2 \mathrm{~kg} /$ $\mathrm{m} 2$, blood pressure was $120 / 60 \mathrm{mmHg}$. Pulse was 68 beats per min. Jugular venous pressure was $5 \mathrm{~cm}$ and heart sounds were normal. A summation gallop was audible and there were no murmurs. Lung fields were clear. There was trace bilateral lower extremity edema, but no hepatomegaly.
Chest radiography showed cardiomegaly and clear lung fields. Repeat echocardiography showed a mildly dilated left ventricle $(5.8 \mathrm{~cm})$ with severe diffuse hypokinesis and an ejection fraction of $25 \%$. The left atrium was mildly dilated. Right ventricular size and wall motion were normal. There was mild-moderate mitral regurgitation, and pulmonary artery systolic pressure was $30 \mathrm{mmHg}$. The transmitral filling pattern was pseudonormal. He walked a distance of $140 \mathrm{~m}$ on a 6 -minute walk test.

\section{Intervention}

The patient participated in supervised 30-minute sessions 2-3 times per week for 6 weeks of ambulation training on an antigravity treadmill (AlterG) (AlterG, Inc., Fremont, CA). Lower body positive pressure (LBPP) was set to $40 \%$ of body weight support. The patient started at a comfortable speed, increasing as tolerated. During his first session, he started and remained at $1 \mathrm{mph}$ for 6.5 minutes and gradually increased to 30 minutes at the end of the 6 weeks. During several of the sessions, the patient reported sudden urgency to urinate, but he tolerated the exercise well without fatigue.

\section{Outcome}

Repeat 6-minute walk test showed an improvement to 540 meters. The patient reported his highest ratings of satisfaction 
and gratitude with the ambulation sessions. His functional class improved to class 2 and he began a walking program. He has remained stable without deterioration at 1-year follow-up.

\section{Discussion}

Congestive heart failure with reduced ejection fraction is a progressive disorder that poses significant morbidity and mortality [1]. In addition to advances in medical and device therapy leading to improved outcomes, exercise remains an overlooked but effective treatment modality shown to reduce hospital admissions and to improve health-related quality of life [2]. However, the unfortunate dilemma is that patients who could potentially derive the most benefit are the least able to be active and engage in exercise. Lower body positive pressure (LBPP) treadmills have recently become widely available to the recreational community. In general, such systems consist of a computer-controlled treadmill equipped with a pressurized air chamber that generates a vertical upward force directly opposing the force of gravity and effectively decreasing body weight [3]. The airtight chamber is formed by the user wearing neoprene shorts that zip around the waist, creating a kayak type skirt from the waist down.

Variable degrees of weight support can be achieved by pumping greater air pressure as WS is proportional to the level of LBPP. In addition, the air-filled chamber improves balance and the frame of the treadmill stabilizes patients with imbalance. Accordingly, antigravity treadmills are used to rehabilitate patients with orthopedic and neuromuscular conditions [4]. A single case report showed improvement in exercise capacity in a patient with morbid obesity following ambulation on an Alter-G treadmill [5]. However, studies evaluating the utility of weight support treadmills in patients with other medical conditions such as CHF are lacking. Accordingly, we report the first case we are aware of in the literature showing improvement of exercise capacity in a patient with severe congestive heart failure. This patient was able to increase 6-minute walk distance by $400 \mathrm{~m}$, which markedly exceeded the $30 \mathrm{~m}$ increase considered significant [6].

He crossed the $300 \mathrm{~m}$ threshold that has prognostic importance [7]. Longer 6-minute walk distance is associated with lower hospitalization rates and higher health related quality of life [2]. The exact mechanism(s) responsible for clinical improvement are unknown but may be related to enhanced oxygen extraction within skeletal muscle microcirculation and better coordinated muscle metabolic control [8]. Also, whereas cardiac autonomic imbalance is deranged during the progression of CHF and dysautonomia improved by exercise, LBPP might portend beneficial effects owing to increased parasympathetic tone. Of note, weight support evokes a number of cardio-respiratory changes as a consequence of increased intra-thoracic blood volume and lower extremity compression, including augmenting venous return, increasing stroke volume and baroreceptor activation [3,9]. The patient remained stable and was able to begin a conventional walking program suggesting that weight supported treadmill ambulation may be useful as a bridge to unsupported exercise.

Our patient's situational urinary urgency might be attributable to increased parasympathetic tone as well as hemodynamic and/ or hydrostatic changes affecting diuresis, analogous to sitting or standing in water [10]. Further studies are required to examine the association of lower body positive pressure and diuresis. Furthermore, he enjoyed the sessions immensely, demonstrating a potential for strong patient satisfaction. In summary, this case report suggests great potential of repeated sessions of ambulation on weight support treadmill to improve functional capacity in patients with medical illnesses such as congestive heart failure by offloading patients' body weight.

\section{References}

1. Bozkurt B (2018) What Is new in heart failure management in 2017? Update on ACC/AHA heart failure guidelines. Curr Cardiol Rep 20(6): 39.

2. Taylor RS, Sagar VA, Davies EJ, Briscoe S, Coats AJ, et al. (2014) Exercisebased rehabilitation for heart failure. Cochrane Database Syst Rev (4): CD003331.

3. Afzal A, Fung D, Galligan S, Godwin EM, Kral JG, et al. (2014) The effect of lower body weight support on arterial wave reflection in healthy adults. J Am Soc Hypertens 8(6): 388-393.

4. Aaslund MK, Helbostad JL, Moe-Nilssen R (2013) Walking during bodyweight-supported treadmill training and acute responses to varying walking speed and body-weight support in ambulatory patients' poststroke. Physiother Theory Pract 29(4): 278-289.

5. Simonson SR, Shimom JM, Long EM, Lester BE (2011) Using the Alter-G treadmill system with an extremely obese female: a case study. Clinical Kinesiology 65(2).

6. Bohannon RW, Crouch R (2017) Minimal clinically important difference for change in 6-minute walk test distance of adults with pathology: a systematic review. J Eval Clin Pract 23(2): 377-381.

7. Pollentier B, Irons SL, Benedetto CM, Dibenedetto AM, Loton D, et al. (2010) Examination of the six-minute walk test to determine functional capacity in people with chronic heart failure: a systematic review. Cardiopulm Phys Ther J 21(1): 13-21.

8. Hirai DM, Musch TI, Poole DC (2015) Exercise training in chronic heart failure: improving skeletal muscle 02 transport and utilization. Am J Physiol Heart Circ Physiol 309(9): H1419-1439.

9. Hsu CY, Hsieh PL, Hsiao SF, Chien MY (2015) Effects of exercise training on autonomic function in chronic heart failure: systematic review. Biomed Res Int 2015: 591708.

10. Wilkins R, Gammage MD, Lewis HM, Bun Tan L, Weissberg PL (1988) Effect of lower body positive pressure on blood pressure, plasma atrial natriuretic factor concentration, and sodium and water excretion in healthy volunteers and cardiac transplant recipients. Cardiovascular research 22(4): 231-235. 


\section{ISSN: 2574-1241}

DOI: $10.26717 / B J S T R .2018 .08 .001621$

Jason M. Lazar. Biomed J Sci \& Tech Res

(c) (P) This work is licensed under Creative

Submission Link: https://biomedres.us/submit-manuscript.php

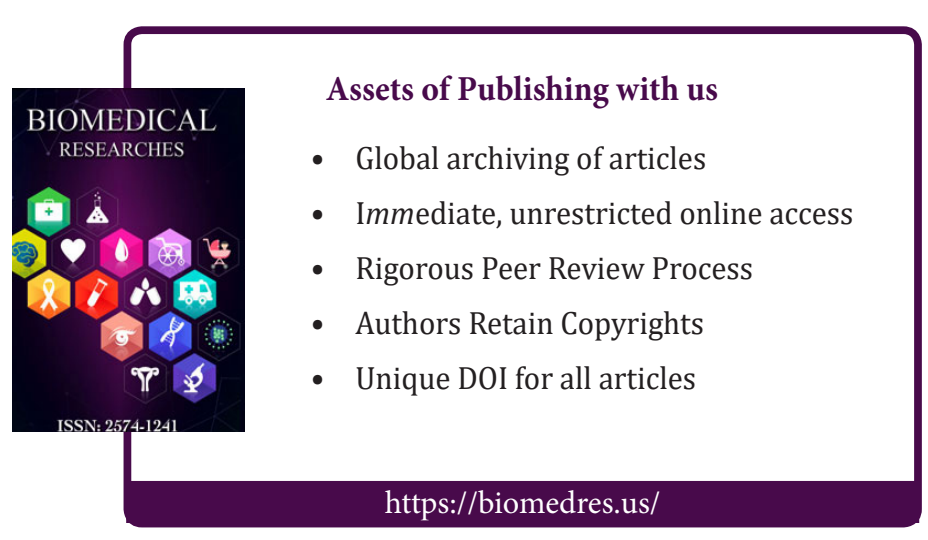

